\title{
Tools for Coordinated Planning Between Observatories
}

\author{
Jeremy Jones ${ }^{a}$, Mark Fishman ${ }^{b}$, Vince Grella ${ }^{b}$, Uri Kerbel ${ }^{b}$, Lori Maks ${ }^{a}$, Dharitri Misra \\ ${ }^{b}$, and Vince Pell ${ }^{\mathrm{b}}$ \\ ${ }^{a}$ NASA Goddard Space Flight Center, Greenbelt, MD 20771 \\ ${ }^{\mathrm{b}}$ CommerceOne Inc., 1100 West Street, Laurel, MD 20707
}

\begin{abstract}
With the realization of NASA's era of great observatories, there are now more than three space-based telescopes operating in different wavebands. This situation provides astronomers with a unique opportunity to simultaneously observe with multiple observatories. Yet scheduling multiple observatories simultaneously is highly inefficient when compared to observations using only one single observatory. Thus, programs using multiple observatories are limited not due to scientific restrictions, but due to operational inefficiencies.
\end{abstract}

At present, multi-observatory programs are conducted by submitting observing proposals separately to each concerned observatory. To assure that the proposed observations can be scheduled, each observatory's staff has to check that the observations are valid and meet all the constraints for their own observatory; in addition, they have to verify that the observations satisfy the constraints of the other observatories. Thus, coordinated observations require painstaking manual collaboration among the observatory staff at each observatory. Due to the lack of automated tools for coordinated observations, this process is time consuming, error-prone, and the outcome of the requests is not certain until the very end. To increase observatory operations efficiency, such manpower intensive processes need to undergo re-engineering.

To overcome this critical deficiency, Goddard Space Flight Center's Advanced Architectures and Automation Branch is developing a prototype effort called the Visual Observation Layout Tool (VOLT). The main objective of the VOLT project is to provide visual tools to help automate the planning of coordinated observations by multiple astronomical observatories, as well as to increase the scheduling probability of all observations.

\section{Introduction}

Planning and executing observations that are coordinated across multiple spacecraft is essential to reaching future space and earth science goals. The current lack of automated tools and interfaces across observatories makes coordinated observing resource intensive for the observatories, and consequently limits scientific research. The proposed tools will facilitate the coordinated observing processes necessary to achieve the concept of a 'virtual observatory', which will spawn a new era of science data collection. 


\section{Objectives and Impact}

\section{Objectives:}

Presently, a number of advanced visual tools are being developed to help the observers and principal investigators of astronomical phenomena in their observation planning. Pioneer among them are the Scientist's Expert Assistant (SEA: http://aaaprod.gsfc.nasa.gov/SEA/) and Astronomer's Proposal Tools (APT: http://ra.stsci.edu/apst/), which enable the observers to simulate the quality of their observation based upon observing parameters (e.g. target properties, instrument setup, and the observatory condition). However, the outcome of the proposed requests is still uncertain as the schedulability of the observation, which is affected by observatory related factors, is still unknown for the requested time period. This problem magnifies many-fold when collaboration among observatories is needed to coordinate the planning of a set of observations through multi-wavelength campaigns - involving time variable phenomena, interacting binary systems, and other events which are of extreme interest to astronomers for quantitative understanding of galactic and extra-galactic phenomena, and for developing realistic physical models.

The main objective of the VOLT project is to provide visual tools to help automate the planning of coordinated observations by multiple astronomical observatories, as well as to increase the scheduling probability of all observations. Thus, these tools will not only provide the users with the required schedulability data, but will also help and guide them in determining the best possible times when the group of observations may be placed in compliance with their coordination goals.

The intended goals of VOLT are:

- The tools will not replicate the software capabilities of the planning/scheduling facility associated with an observatory. Rather, they will interface with these software components, using state-of-the-art communication mechanisms, and retrieve the desired data. Required formatting and normalization of data may be performed by these tools if necessary.

- The coordination of observations will be modeled as a network of temporal constraints, which can be solved in a satisfactory manner by the use of an appropriate constraint satisfaction engine. We will provide cost-effective solutions by using free or low-cost search engines that will be adequate for our purpose.

- We will provide the users with explanatory help on constraints and constraint relaxation suggestions to make an observation/coordination schedulable if and when it fails to be so.

- We will emphasize reuse and modularity by making our tools easily extensible and configurable to new missions. Although we will initially apply it to space-based observatories, later we will expand to queue-based ground observatories, other space sciences missions, as well as to other domains such as earth sciences missions. 


\section{Impact:}

The primary impact of the tools provided by VOLT will be in the arena of coordinated observations, involving two or more observatories, as shown below:

1) Coordinated Observations are limited by observatories because of the complexity of the planning process, and the manpower and manual effort required for coordination. VOLT seeks to automate this planning; to enable new types of complex, coordinated observations that are not feasible with current manual methods, and to make coordinated observing a low cost, routine task rather than a special exception.

Although multi-observatory coordinations involving three to five observatories are envisioned for some studies, currently very few coordinated observations are requested $(7-10 \%$ of observations for $\mathrm{HST})$ and take place in these observatories due to the uncertain, manual and labor-intensive nature of such coordinations. Due to the lack of manpower resources, some observatories such as FUSE do not support coordinated planning except under extreme cases. Users themselves are also discouraged from requesting three or more coordinated observations due to the complexity involved. Our tools will help both the astronomers and observatory scheduling staff in planning for such complex observations. They will reduce the manual workload, and thus the cost, of coordinated and timeconstrained observations.

2) Observers of coordinated observing programs hardly have any tools that will allow them to plan their observations effectively. VOLT will fill this void by applying new technology and innovative solutions for this increasingly important aspect of science planning.

VOLT will help the observation planning not only by automating the procedure, but also providing visual cues on coordination, and allowing the users to look for alternate solutions. It addresses the problem by applying new technology and innovative solutions. By helping in the planning of observations these tools will help in generating more feasible requests even for a single observatory. The pluggable nature of these tools (into other proposal/observation planning tool sets such as APT) will provide an integrated environment enhancing the observation's scheduling probability. The net result will be an increase in science data that would help in the understanding of galactic and extra-galactic phenomena, and benefit a wide variety of astronomical research.

3) By achieving 1 and 2, more astronomers will apply for coordinated observations, making coordinated observations seem so standard that observatories can take the next step to coordination - one proposal for all observatories involved in the coordinated program so that a given scientific topic can be investigated as a whole rather than the observer hoping that the proposal will succeed for each observatory separately. 
Figure 1: The VOLT prototype schedulability display

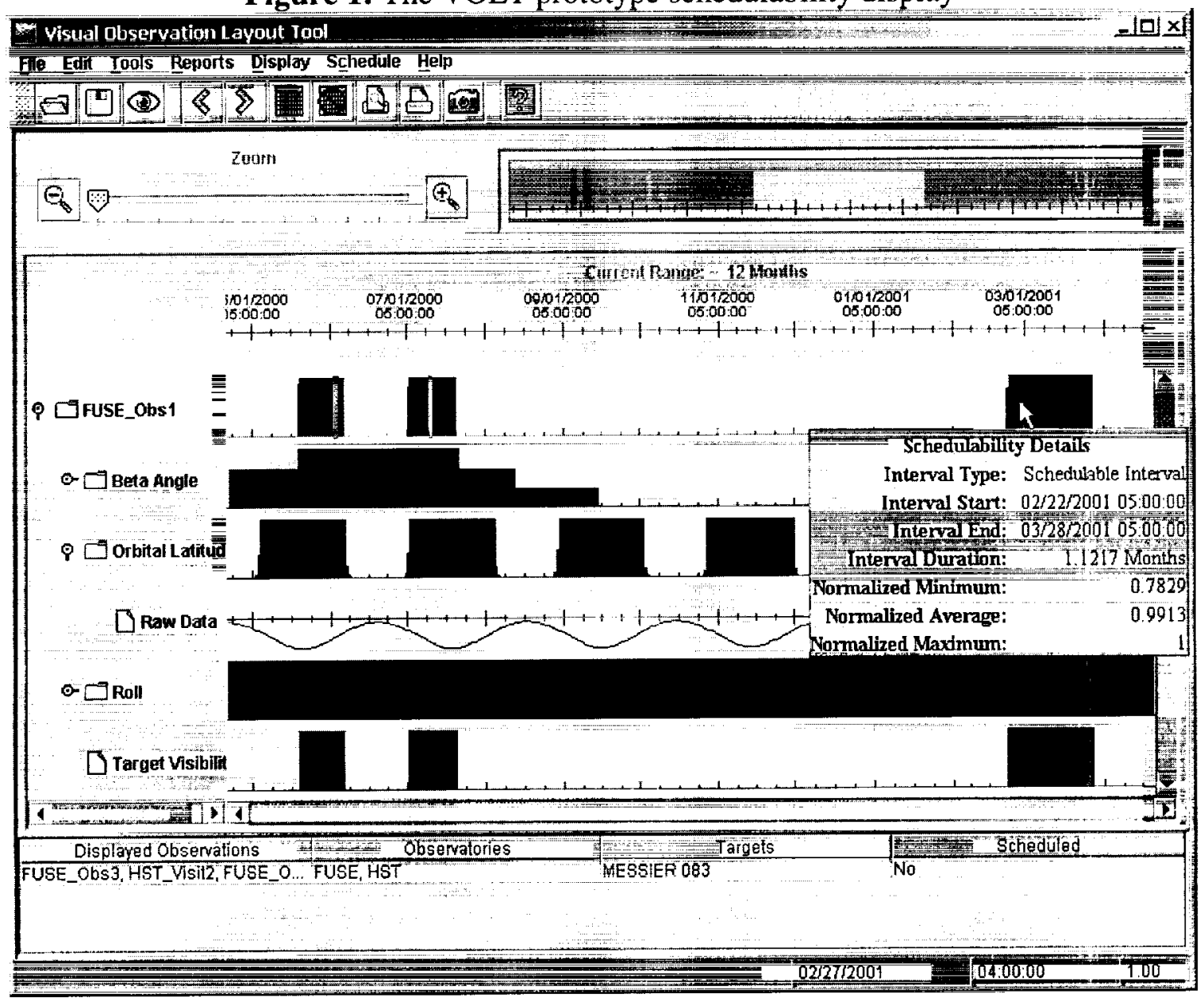

\section{Acknowledgments}

The authors would like to acknowledge the active support of the following persons in developing the VOLT prototype:

Glenn Miller, Beth Perriello, Anuradha Koratkar, Karla Peterson and Peg Stanley: Space Telescope Science Institute - for help in establishing VOLT system requirements. Bryce Roberts: Johns Hopkins University - for help in interfacing with FUSE observatory data.

\section{References}

"Coordinating multi-wavelength campaigns between observatories", Karla Peterson, Marty Eckert, Nancy Remage Evans, Paul Hilton, Beth Perriello, Bryce Roberts, Evan Smith, Peg Stanley, Observatory Operations to Optimize Scientific Return II, Proceedings of SPIE, Volume 4010, Editor: P. Quinn, pp 279-289.

"Streamlining Collaborative Planning in Spacecraft Mission Architectures", Dharitri Misra, Mike Bopf, Mark Fishman, Jeremy Jones, Uri Kerbel, Vince Pell, SpaceOps2000. 